\title{
Huge metastatic multicystic ovarian cancer with liver involvement: A case Report
}

\author{
Ngim, Ogbu Ewezu ${ }^{1, ~ *, ~ A g o, ~ B o n i f a c e ~ U j i i, ~ ", ~ O g b u d u ~ S t e p h e n ~}{ }^{3}$, Nwagboso Chima ${ }^{3}$, \\ Echei Chidiebere $^{3}$, Etiuma, Anietimfom Umoh ${ }^{3}$, Bassey, Okon Odokwo ${ }^{3}$, Ndoma-Egba Rowland ${ }^{1}$ \\ ${ }^{1}$ Division of Hepatopancreatobiliary Surgery, Department of Surgery, University of Calabar and University of Calabar Teaching Hospital , \\ Calabar, Cross River State, Nigeria \\ ${ }^{2}$ Gynaecological Oncology Firm, Department of Obstetrics and Gynaecology, University of Calabar and University of Calabar Teaching \\ Hospital, Calabar, Cross River State, Nigeria \\ ${ }^{3}$ Division of Cardiothoracic Surgery, Department of Surgery, University of Calabar and University of Calabar Teaching Hospital , \\ Calabar, Cross River State, Nigeria
}

\section{Email address:}

ogbungim@yahoo.com (Ngim, Ogbu E), bonifaceago@yahoo.com (Ago, B. U)

\section{To cite this article:}

Ngim, Ogbu Ewezu, Ago, Boniface Uji, Ogbudu Stephen, Nwagboso Chima, Echei Chidiebere, Etiuma, Anietimfom Umoh, Bassey, Okon Odokwo, Ndoma-Egba Rowland. Huge Metastatic Multicystic Ovarian Cancer with Liver Involvement: A Case Report. Journal of Cancer Treatment and Research. Vol. 2, No. 3, 2014, pp. 21-26. doi: 10.11648/j.jctr.20140203.11

\begin{abstract}
Introduction: Ovarian cancers are gynaecological malignancies described as the most lethal because they often present at the advanced stages. Metastasis to the liver parenchyma occurs in stage IVB disease. The diaphram is a tumour sanctuary site as most deposits are missed during cytoreductive surgeries and chemotherapy. Materials and method: This is a case presentation of a 39 year old lady with recurrent huge multicystic ovarian cancer with liver and diaphragmatic involvement. Abdominal ultrasound scan and a CT-scan were done amongst other investigations. This patient was comanaged by the Hepato-Pancreato-Biliary surgeons, Cardiothoracic Surgeons and the Gynaecologic oncologist. She had cytoreductive surgery and adjuvant chemotherapy. Conclusion: Diaphragmatic involvement from ovarian tumour is rare and represents an advanced disease. The superiority of CT-scan over abdominal ultrasound scan in this case is incontrovertible. A multidisciplinary approach is an important cornerstone in it's management.
\end{abstract}

Keywords: Ovarian Cancers, Liver and Diaphragmatic Involvement, CT-Scan

\section{Introduction}

Liver involvement in ovarian cancer is not uncommon and often indicates an advanced disease ${ }^{1-3}$. Radiological imaging modalities are often indispensable in making a diagnosis. Abdominal CT scan has proven to be superior to abdominal ultrasound scan in making a diagnosis and delineating the extent of metastasis. In developing countries, CT-scan is still relatively unavailable in most centres thus making adequate evaluation of these patients an almost impossible task ${ }^{3}$. This impacts negatively on their treatment and follow-up. Nulliparity and age above 55 years are known risk factors. In our environment, these tumours are seen at a younger age and the prognosis is generally poor. A multidisciplinary approach is often the rule in managing these patients ${ }^{1-5}$.

\section{Case Presentation}

Mrs UH, a 39 year old nuliparous lady was referred to the gynaecologic oncology unit of the University of Calabar Teaching Hospital (UCTH) from a private hospital in Aba, Nigeria, due to a large abdominal mass and gross ascites. She had staging laparotomy and optimal debulking followed by adjuvant chemotherapy. It was a stage IVB disease histologically confirmed to be serous adenocarcinoma. She had uneventful postoperative period and tolerated the chemotherapy well.

She had no fresh complaints until two years after surgery when she noticed that for two months prior to presentation, she had had an increase in her adominal girth associated with right sided abdominal pain, and generalised malaise. 
Abdominopelvic ultrasonography showed a cyst in the liver as well as another in the pelvis. She was then referred to the Hepatopancreatobiliary Surgery unit of the University of Calabar Teaching Hospital (UCTH).

An abdominal CT scan done revealed a large multicystic lesion attached to the liver with subdiaphramatic involvement. At surgery, a huge multicystic tumour was found involving the whole of the right side of the abdomen from pelvis to the diaphragm. Also at surgery, a right intercostal drain was put in place because of a rent in the diaphragm while debulking the tumour. The tumour could not be completely removed because of the extent of spread at the time of surgery. Patient had an uneventful postoperative recovery and had four cycles of chemotherapy by the gynaecologist after the secondary surgery.

The patient is still on follow-up with Karnofsky performance status $90-100$.

Figures 1-6 shows the CT-scan and operative pictures of the patient.

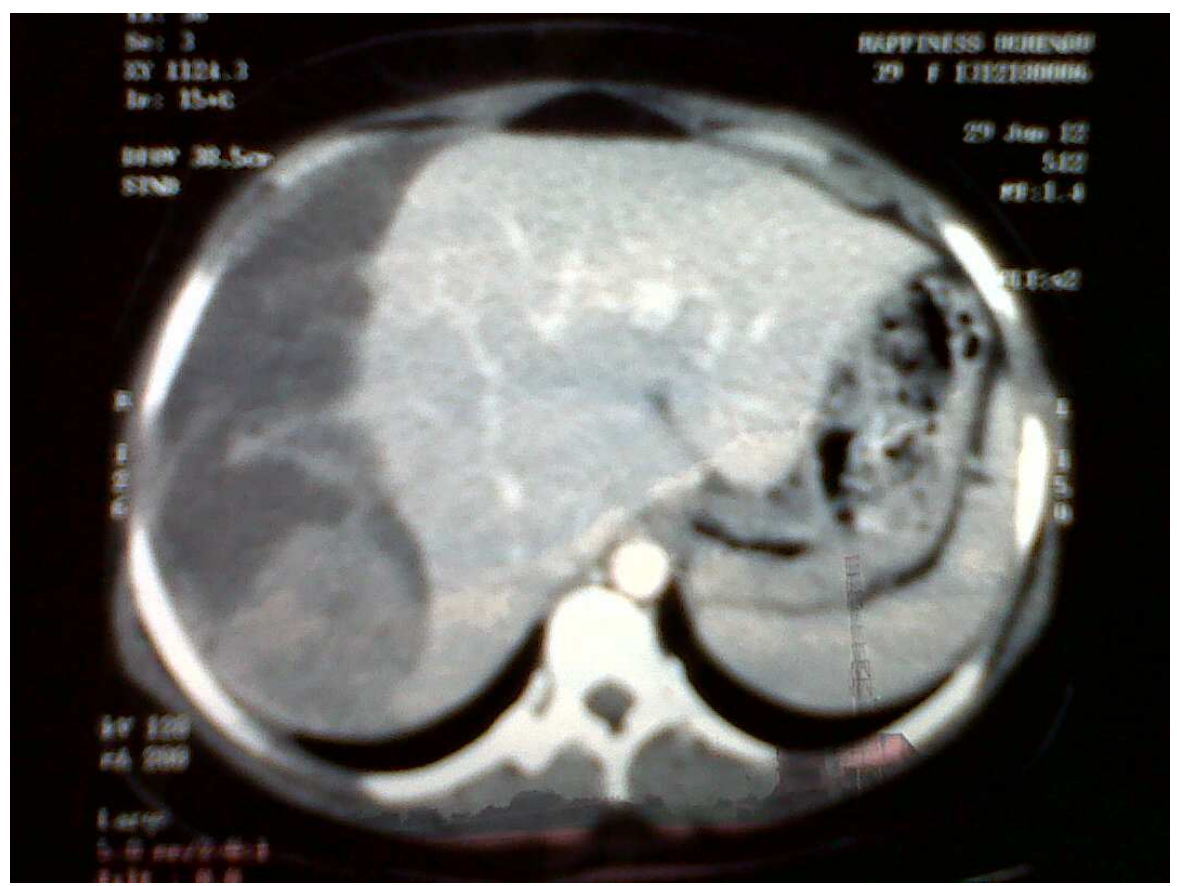

Figure 1. CT Scan picture showing a multicystic mass pushing the liver medially

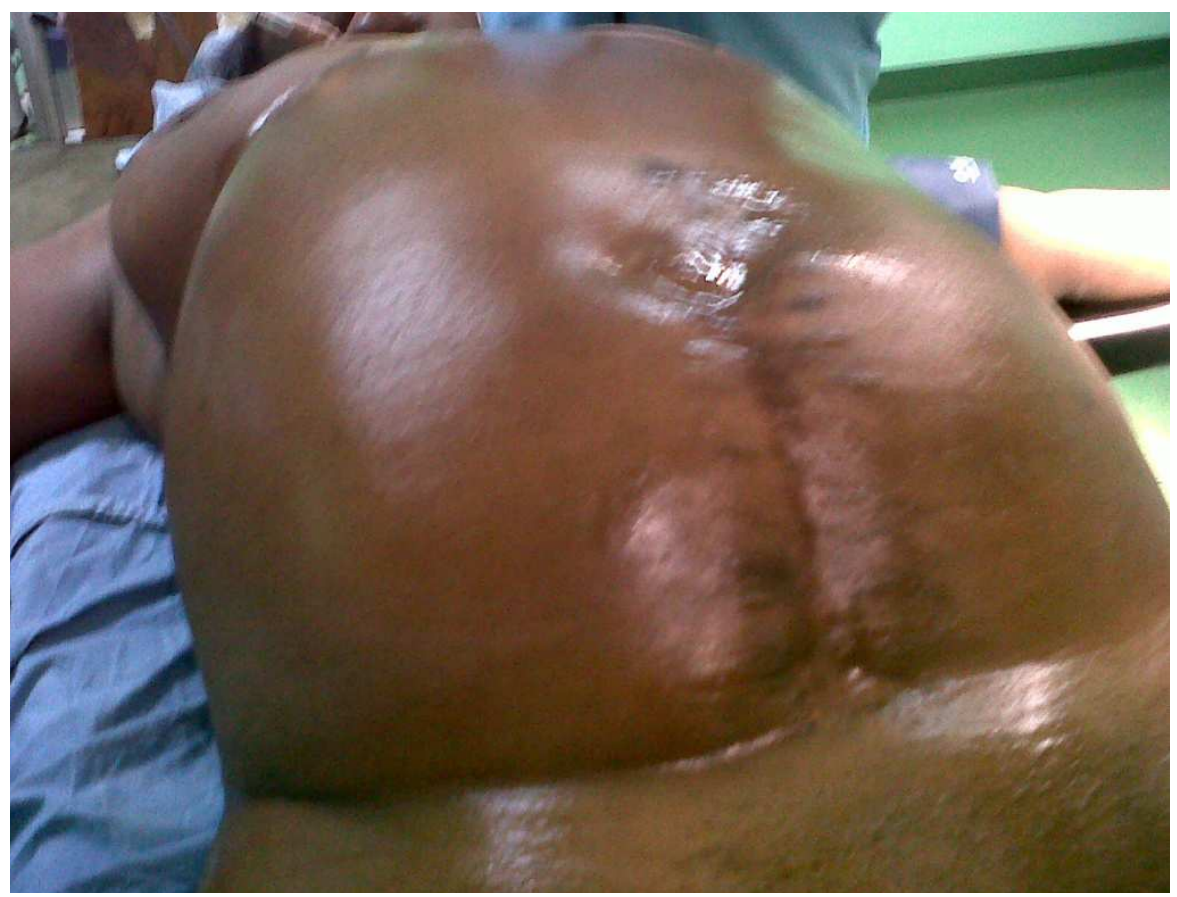

Figure 2. Shows the patient on the operating table with a huge right sided abdominal swelling and previous laparotomy scar from the initial surgery by the gynaecologist. 


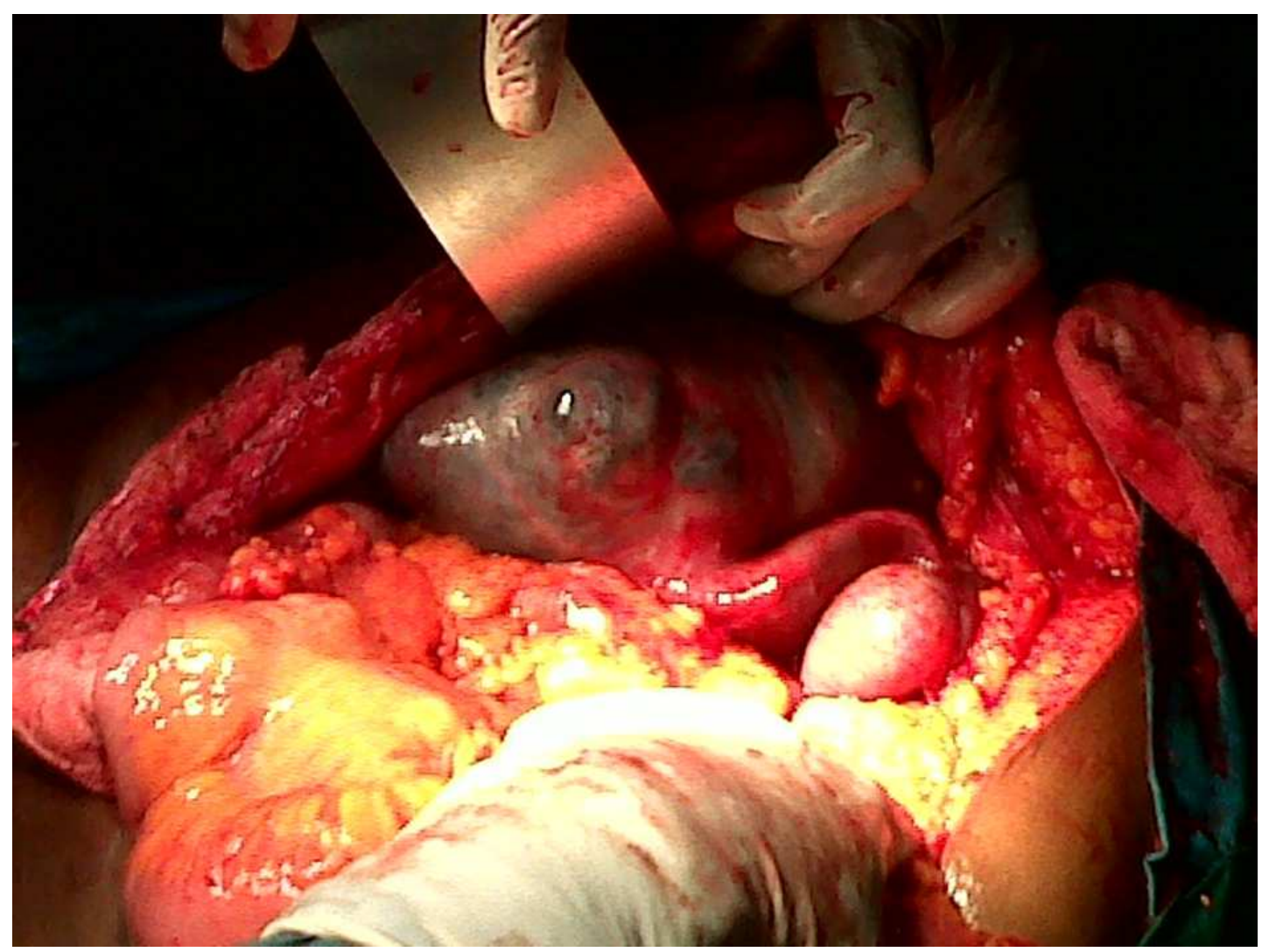

Figures 3. Shows a multicystic ovarian tumour attached to the liver

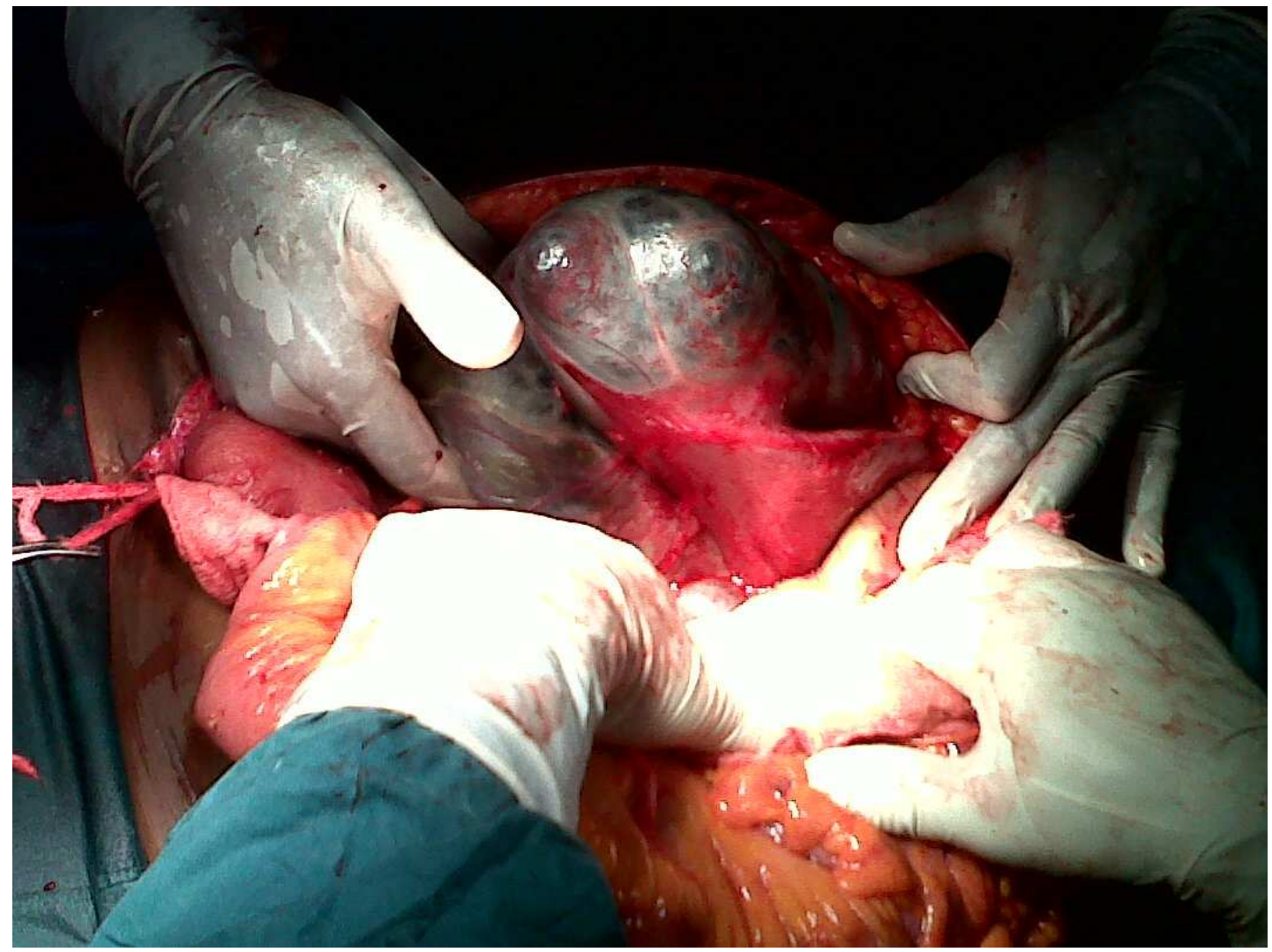

Figure 4. Shows more of the huge multicystic ovarian tumour attached to the liver 


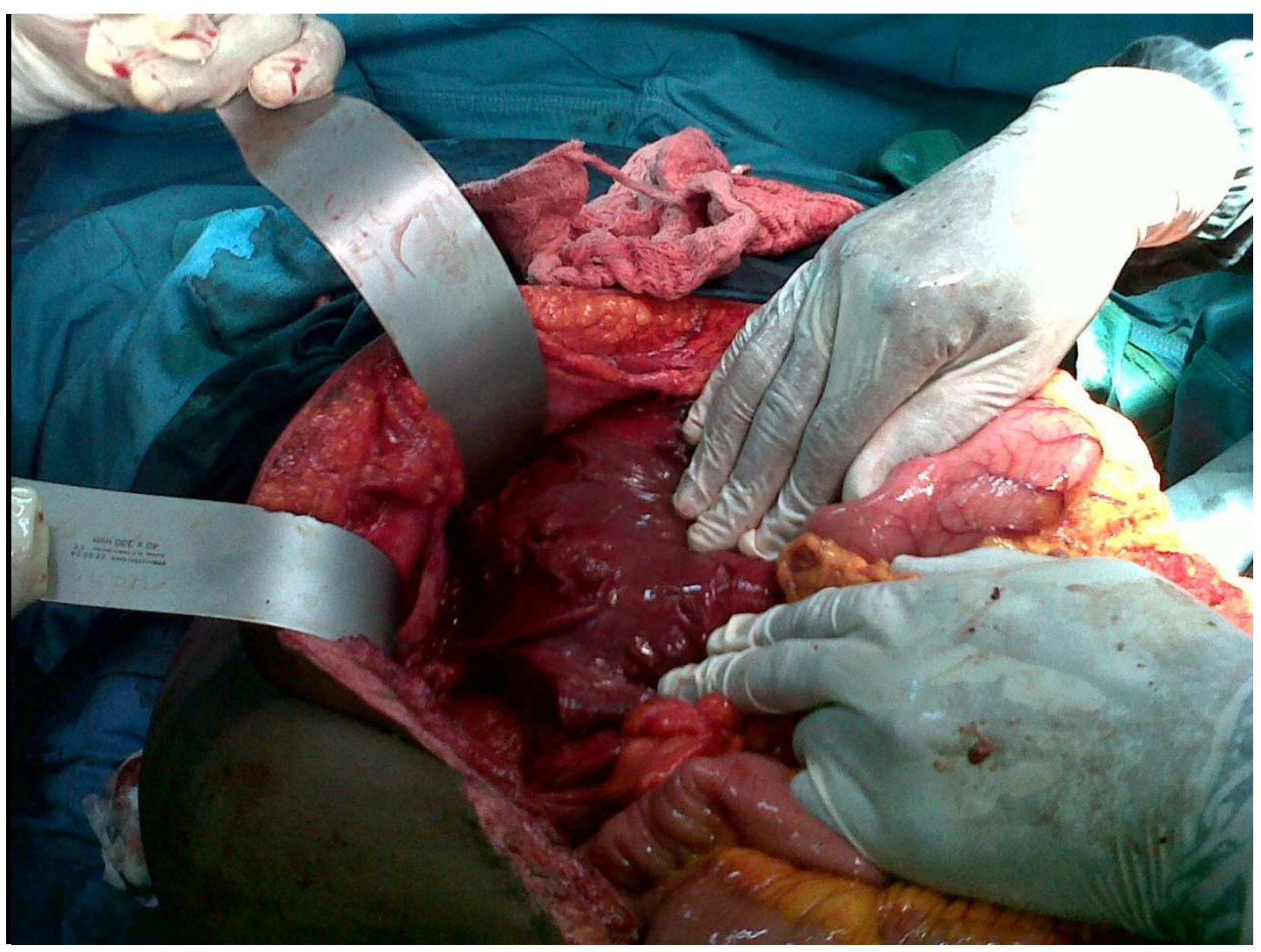

Figure 5. Shows the denuded surface of the liver after debulking the tumour

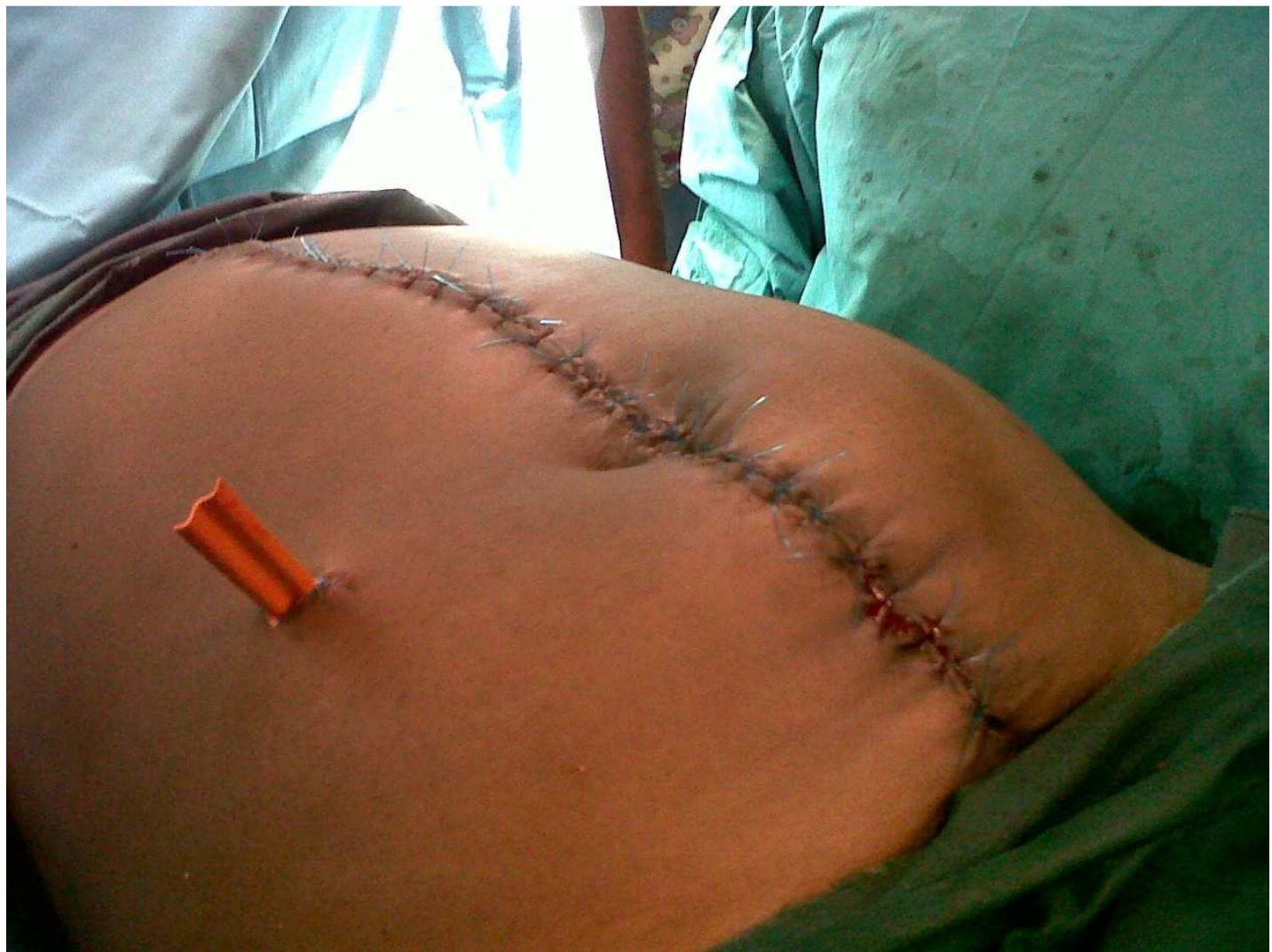

Figure 6. Shows of the abdomen at the end of surgery with a corrugated rubber drain placed in the abdominal cavity. 


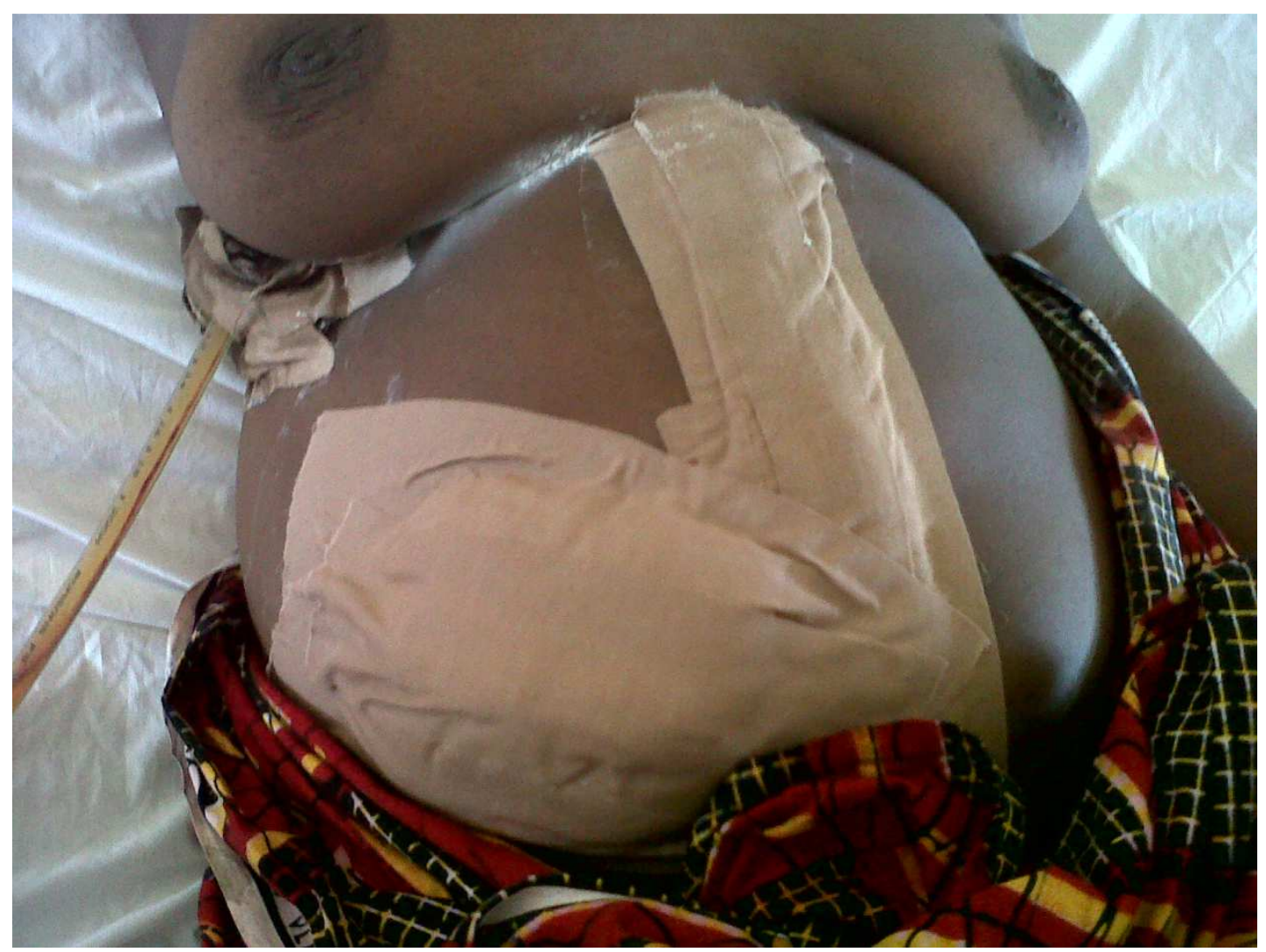

Figure 7. Shows a right chest tube for the closed thoracostomy drainage

\section{Discussion}

The Surveillance, Epidemiology, and End Result program (SEER) of the US National Cancer Institute 2013, showed that there is a $1.4 \%$ increased risk of a woman developing ovarian cancer at some point in her lifetime ${ }^{1}$. What is most worrisome is that early symptoms may be missed as most women present at the advanced stages of the disease where radical tumour debulking procedures to obtain optimal residual (tumour nodule no larger than $1 \mathrm{~cm}$ ), can be quite challenging ${ }^{2}$. This may have been the case for this patient where the diaphragmatic implants were missed at the primary surgery and even the adjuvant chemotherapy could not eradicate them.

In Africa as in many other countries, the incidence of this condition is on the increase and many of these patients present with late disease. Surgical staging of ovarian cancer is based on the International Federation of Gynecology and Obstetrics (FIGO) staging system. Stage I ovarian cancer is confined to the ovaries. In stage II ovarian cancer there is peritoneal metastases confined to the true pelvis. Stage III ovarian cancer refers to a tumor with extrapelvic peritoneal metastases or abdominopelvic nodal metastases while stage IV refers to ovarian cancer with distant metastases, such as malignant pleural effusion and parenchymal liver metastases ${ }^{1}$. Sometimes, the liver parenchyma can be invaded by perihepatic metastasis. Differentiation of perihepatic metastases with liver parenchymal invasion from those without invasion is important for the selection of an appropriate surgical approach. Perihepatic metastases in advanced ovarian cancer, occur by means of peritoneal spread of tumor implants on the liver surface with invasion of the liver parenchyma in some cases ${ }^{3}$

Our index patient clearly had a stage IV disease from a recurrent ovarian tumour. Abdominal CT scan proved its superiority over ultrasound scan in making a diagnosis in this patient thus aiding intervention. The liver and diaphragmatic involvement made this case unique, hence this report. Edwards et $\mathrm{al}^{4}$ reported a case of recurrent papillary serous carcinoma of the ovary involving the liver parenchyma through the full thickness of the diaphragm into the lung parenchyma. Their patient had cytoreductive surgery by a multidisciplinary team just like in our index patient. They noted that the tumour could not be completely removed because of the stage which is a similar scenario in our index patient. Though multiple debulking procedures for ovarian cancer are advocated by some authorities, its usefulness is controversial, especially when there is extensive upper abdominal orS thoracic disease ${ }^{4}$. However, optimal surgical debulking and performance status have been shown to be important determinants of survival in patients with Stage IV epithelial ovarian cancer. Also,even in patients with unresectable liver metastasis, optimal debulking of extrahepatic disease is associated with a significant survival advantage ${ }^{6-8}$. Furthermore, these debulking surgeries, also address any pressure symptoms posed by the shear size of the tumour like in our index patient. Our patient had relief from the abdominal pain 
which she had been experiencing before the surgery. Hepatic resection of metachronous metastases from gynecologic carcinomas can be performed safely and may help prolong survival in carefully selected patients ${ }^{9}$. Many authors have emphasized the need for a multidisciplinary approach to the management of this condition ${ }^{2-11}$. Overall, findings by Lim et $\mathrm{al}^{12}$ and some other workers ${ }^{3-8}$ suggest that complete hepatic resection should be attempted for patients with hepatic parenchymal metastasis from peritoneal seeding as this is said to prolong survival in stage IV epithelial ovarian cancer.

\section{Conclusion}

Extra-pelvic involvement of ovarian cancer is a common feature of stage IV ovarian cancer. Appropriate clinical assessment with imaging are essential in making a diagnosis. A multidisciplinary cytoreductive surgery and adjuvant chemotherapy are keys to successful management of these patients.

\section{References}

[1] SEER. Cancer statistics, $\mathrm{http} \cdot / /$ seer.cancer gov/statistics $/ \mathrm{html} /$ ovary $/ \mathrm{html}$ 14th March 2014

[2] Nashmia JAM, Tien L. Does modality of adjuvant chemotherapy after interval surgical debulking matter in epithelial ovarian cancer. IJGC 2014; 24(3): 461-467

[3] Oguz Akin, Evis Sala, Chaya S Moskowitz,Nicole Ishill ,Richard A Soslow, Dennis S Chi , Hedvig Hricak . Perihepatic metastasis from ovarian cancer:Sensitivity and specificity of CT for the detection of metastasis with and those without liver parenchymal invasion. Radiology 2008 248:511-517.

[4] Edwards JM, Lowery WJ, Secord AA. Primary debulking for epithelial ovarian cancer. JSCR ,2012 7:7

[5] Bosquet JG, Merideth MA, Podratz KC, Nagorny DM .Hepatic resection for metachronous metastasis from ovarian carcinoma . HPB (Oxford), 2006 April 1; 8(2):

[6] Bristow RE, Montz FJ, Lagasse LD, Leuchter RS, Karlan BY. Survival impact of surgical cytoreduction in stage IV epithelial ovarian cancer. Gynecol Oncol. 1999 Mar;72(3):278-87.

[7] Scarabelli C, Gallo A, Carbone A. Secondary cytoreductive surgery for patients with recurrent epithelial ovarian carcinoma. Gynecol Oncol. 2001 Dec;83(3):504-12.

[8] Jose M. Ramia*, Roberto De La Plaza, Jose Quiñones, Pilar Veguillas, Farah Adel, Jorge García-Parreño. Liver Metastases from Gynecological Cancers: Time to Resection? Surgical Science, 2012, 3, 120-125

[9] Merideth MA, Cliby WA, Keeney GL, Lesnick TG, Nagorney DM, Podratz KC. Hepatic resection for metachronous metastases from ovarian carcinoma. Gynecol Oncol. 2003 Apr;89(1):16-21.

[10] Guang-cai Niu, Chang-ming Shen, Wei Cui, Qiang Li. Hepatic Resection is Safe for Metachronous Hepatic Metastases from Ovarian Cancer. Cancer Biol Med. 2012 September; 9(3): 182-187

[11] Hepatic resection for metastatic gynecologic carcinomas.Gynecol Oncol. 1997 Jul;66(1):45-51.Chi DS, Fong Y, Venkatraman ES, Barakat RR.

[12] Lim MC, Kang S, Lee KS, Han SS, Park SJ, Seo SS, Park SY. The clinical significance of hepatic parenchymal metastasis in patients with primary epithelial ovarian cancer. Gynecol Oncol. 2009 Jan;112(1):28-34. 